\title{
ESTRESSES HÍDRICO E SALINO NO PROCESSO GERMINATIVO DAS SEMENTES DE Plantago ovata Forsk. (Plantaginaceae) ${ }^{1}$
}

\author{
Marcílio Pereira Sousa², Lúcia Filgueiras Braga², João Filgueiras Braga² e Maria Elena Aparecida \\ Delachiave $^{2}$
}

\begin{abstract}
RESUMO - Plantago ovata é espécie medicinal de grande importância cultivada na Índia, no Irã e no Paquistão. As sementes possuem de 20 a 30\% de mucilagem utilizada pelas indústrias farmacêuticas nos tratamentos de constipação crônica, câncer intestinal e amebíase. A Índia é o principal exportador mundial das sementes de Plantago ovata, tendo o produto comercial com essas sementes, no Brasil, o nome de Agiolax. O objetivo deste trabalho foi avaliar os possíveis efeitos dos estresses hídrico e salino na germinação de sementes de Plantago ovata. As sementes foram colocadas para germinar em papel umedecido com $15 \mathrm{~mL}$ de soluções nos potenciais osmóticos de 0,$0 ;-0,2 ;-0,4 ;-0,6$; e -0,8 MPa, induzidos com polietileno glicol (PEG 6000), $\mathrm{NaCl}$ e $\mathrm{CaCl}_{2}$. O experimento constou de quatro repetições com 50 sementes cada, na temperatura de $25^{\circ} \mathrm{C}$, na presença de luz. A análise dos resultados permitiu a conclusão de que o estresse hídrico acarreta maior redução na porcentagem e velocidade de germinação das sementes de $P$. ovata do que o estresse salino, e, independentemente da substância utilizada para indução do estresse, o limite para germinação está entre -0,4 e 0,6 MPa.
\end{abstract}

Palavras-chave: Estresses hídrico e salino, Plantago ovata e germinação.

\section{WATER AND SALINE STRESSES ON THE GERMINATION OF Plantago ovata Forsk. (Plantaginaceae) SEEDS}

\begin{abstract}
Plantago ovata is a medicinal species of great importance cultivated in India, Iran and Pakistan.The seeds contain 20 to $30 \%$ of mucilage, used by the pharmaceutical industry for the treatment of chronic constipation, intestinal cancer and amoeba. India is the main exporter of Plantago ovata seeds, worldwide. In Brazil, this species has been given the corporate name of Agiolax. This work aimed to evaluate the possible effects of water and salt stresses on the germination of Plantago ovata seeds. The seeds were put to germinate in paper moistened with $15 \mathrm{~mL}$ of solutions with the potentials of $0.0 ;-0.2 ;-0.4 ;-0.6$ and $-0.8 \mathrm{MPa}$, induced with polyethylene glycol (PEG 6000), $\mathrm{NaCl}$ and CaCl2. Each experiment consisted of 50 seeds, 4 repetitions, and temperature at $25^{\circ} \mathrm{C}$ in the presence of light. The results allowed the conclusion that water stress causes a greater reduction in the percentage and speed of germination of $\boldsymbol{P}$. ovata seeds than saline stress, regardless of the substance used for stress induction, germination limit was-0.4 to -0.6MPa.
\end{abstract}

Keywords: Water and saline stresses, Plantago ovata and germination.

\footnotetext{
${ }^{1}$ Recebido em 23.08.2006 e aceito para publicação em 15.12.2007.

${ }^{2}$ Universidade do Estado do Mato Grosso (UNEMAT), Campus de Alta Floresta, Rod. MT Km 147, Jardim Tropical, 78580-000 Alta Floresta-MT. E-mail : >marcilio.sousa@pq.cnpq.br>e <luciabraga@unemat.br>.
} 


\section{INTRODUÇÃO}

Plantago ovata é uma espécie medicinal de grande importância cultivada na Índia, Irã e Paquistão, devido ao fato de as sementes possuírem de 20 a 30\% de mucilagem, utilizada pelas indústrias farmacêuticas na produção de medicamentos para tratamento de constipação crônica, câncer intestinal, amebíase, disenteria e irritações gastrointestinais. A Índia é o principal exportador mundial das sementes de $P$. ovata, e no Brasil o produto comercial composto por essas sementes recebe o nome de Agiolax.

De acordo com Lima et al. (2006), não há conhecimento suficiente para o manejo e análise de sementes da maioria das espécies, de modo a fornecer dados que possam caracterizar seus atributos físicos e fisiológicos. Nesse contexto, Larcher (2000) salientou que, no estudo da germinação de sementes, o conhecimento sobre como o estresse afeta esse processo tem importância especial na ecofisiologia para avaliação dos limites de tolerância e da capacidade de adaptação das espécies, pois os fatores abióticos interferem na germinação de sementes.

Dentre esses fatores, a água é de extrema importância, pois, de acordo com Mayer e Poljakoff-Mayber (1989) e Carvalho e Nakagawa (2000), ela não é apenas o primeiro fator que inicia a germinação, mas também está envolvida, direta ou indiretamente nesse processo. Em todas as demais etapas do metabolismo subseqüente tem havido a sua participação decisiva nas reações enzimáticas e na solubilização e transporte de metabólitos e como reagente na digestão hidrolítica de proteínas, carboidratos e lipídeos dos tecidos de reserva da semente.

Além da água, a salinidade do solo influencia significativamente a resposta germinativa. O excesso de sais solúveis provoca redução no potencial hídrico do solo, refletindo menor capacidade de absorção de água pelas sementes. Os efeitos dos sais solúveis se manifestam através da pressão osmótica elevada e da ação tóxica de alguns elementos, como o sódio e o cloro, que promovem distúrbios fisiológicos na planta e podem ser letais (SILVA et al., 1992; BRACCINI et al., 1996).

As plantas com baixa tolerância à salinidade nos vários estádios de desenvolvimento, incluindo a germinação, são denominadas glicófitas e as mais tolerantes, halófitas (MAYER e POLJAKOFF-MAYBER, 1989; LARCHER, 2000).
De modo geral, soluções salinizadas são reportadas como tendo efeito inibidor superior ao das soluções osmóticas não tóxicas de potencial equivalente (KHATRI et al., 1991).

Um dos métodos mais difundidos para determinação da tolerância das plantas ao excesso de sais é a observação da porcentagem de germinação em substratos salinos. A redução do poder germinativo, em comparação com o controle, serve como indicador do índice de tolerância da espécie à salinidade e aos estádios subseqüentes do desenvolvimento (SILVA et al., 1992). Bradford (1995) e Braccini et al. (1996) afirmaram que a salinidade afeta a germinação não só dificultando a cinética de absorção da água, mas também facilitando a entrada de íons em quantidade tóxica nas sementes em embebição, enquanto Santos et al. (1992) relataram, a respeito de sementes submetidas ao estresse hídrico, que a diminuição na germinação é atribuída à redução da atividade enzimática, a qual promove menor desenvolvimento meristemático. Campos e Assunção (1990) atribuíram maior redução da germinação de sementes de arroz pelos sais $\mathrm{NaCl}$ e $\mathrm{Na}_{2} \mathrm{SO}_{4}$, quando comparados com polietileno glicol 6000, a uma aparente inibição da síntese e, ou, atividade de enzimas hidrolíticas necessárias à germinação, provocadas pelos sais em altas concentrações. Tobe et al. (2004), estudando os efeitos de cinco sais, verificaram que os tratamentos utilizando $\mathrm{NaCl}$ e $\mathrm{MgCl}_{2}$ e a adição de $\mathrm{CaCl}_{2}$ em baixas concentrações favoreceram germinação das sementes Haloxylon ammodendron, e as respostas em relação ao crescimento inicial da espécie nas soluções salinas foram atribuídas às diferenças entre componentes salinos e seus efeitos tóxicos na permeabilidade das membranas celulares. Martin e Trine (2005), estudando as espécies Plantago maritima, Juncus gerardii, Artemisia vulgaris, Agrostis spp. e Rumex spp., verificaram que houve redução na porcentagem e velocidade de germinação entre 200 e $400 \mathrm{mM}$ de $\mathrm{NaCl}$.

Devido à importância medicinal e econômica e à escassez de estudos sobre a germinação de sementes de Plantago ovata Forsk., este trabalho teve como objetivo verificar a tolerância aos estresses hídrico e salino no processo germinativo dessa espécie.

\section{MATERIAL E MÉTODOS}

O trabalho foi conduzido nos laboratórios do Departamento de Botânica, do Instituto de Biociências - UNESP - Campus de Botucatu. As sementes de 
Plantago ovata foram doadas pela Empresa Centroflora Anidro, oriundas do Canadá, sob o número do lote 043214, com 98\% de germinação.

Para determinar o grau de umidade inicial, foram utilizadas duas amostras de 25 sementes cada, as quais foram pesadas e colocadas em estufa a $105 \pm 3^{\circ} \mathrm{C}$ por 24 h e pesadas novamente, procedendo-se ao cálculo do grau de umidade das sementes, com base no peso úmido, conforme procedimento descrito por Brasil (1992), apresentando $6 \%$ de umidade. Durante a fase experimental, as sementes permaneceram armazenadas por um ano em potes de vidro, fechados e colocados em geladeira.

Para verificar o efeito de diferentes potenciais hídricos no processo germinativo, foi utilizado como agente osmótico o polietileno glicol (PEG 6000), sendo as soluções preparadas segundo a tabela citada por Villela et al. (1991). Para simular o estresse salino, foram empregados os sais $\mathrm{NaCl}$ e $\mathrm{CaCl}_{2}$, sendo as soluções salinas preparadas a partir da equação de Van’t Hoff, citada por Salisbury e Ross (1992): $\psi_{\text {os }}=-$ RTC, em que:

$\psi_{\text {os }}$ potencial osmótico $($ atm $)$;

$\mathrm{R}=$ constante geral dos gases perfeitos $(0,082$ atm $\left.\cdot 1 \cdot \mathrm{mol}^{10} \mathrm{k}^{-1}\right)$;

$$
\begin{aligned}
& \mathrm{T}=\text { temperatura }\left({ }^{\circ} \mathrm{K}\right) ; \mathrm{e} \\
& \mathrm{C}=\text { concentração }(\mathrm{mol} / 1)\left(\mathrm{N}^{0}\right. \text { de moles/l). }
\end{aligned}
$$

Para tanto, foram utilizadas quatro repetições com 50 sementes, colocadas para germinar em caixas tipo Gerbox transparentes, forradas com papel-filtro umedecido com $15 \mathrm{~mL}$ das diferentes soluções de PEG, $\mathrm{NaCl}$ e $\mathrm{CaCl}_{2}$, nos potenciais de 0,0 (testemunha) -0,2; -0,4; -0,6; e -0,8 MPa, à temperatura de $25^{\circ} \mathrm{C}$, na presença de luz, com quatro lâmpadas fluorescentes brancas, tipo luz do dia, com irradiância média de 320W.cm².

O delineamento experimental foi o inteiramente casualizado contendo cinco potenciais e três agentes osmóticos, totalizando 15 tratamentos com quatro repetições, sendo a comparação de médias realizada pelo teste de Tukey a 5\% de probabilidade.

As leituras de germinação foram realizadas diariamente, sendo consideradas germinadas as sementes que apresentaram extensão radicular igual ou superior a $2 \mathrm{~mm}$ (REHMAN et al., 1996), as quais foram retiradas
(BRASIL, 1992). Os cálculos de porcentagem e velocidade de germinação foram realizados de acordo com Labouriau e Valadares (1976) e Maguire (1962), respectivamente.

$$
G(\%)=\frac{N}{A} \cdot 100
$$

em que: $\mathrm{N}=$ número de sementes germinadas; e

$A=$ número total de sementes colocadas para germinar.

$$
I V G=\frac{N_{1}}{D_{1}}+\frac{N_{2}}{D_{2}}+\ldots . .+\frac{N_{n}}{D_{n}}
$$

em que: IVG = Índice de Velocidade de Germinação; $\mathrm{n}$; e

$\mathrm{N}_{1: \mathrm{n}}=$ número de plântulas germinadas no dia $1, \ldots .$.

$\mathrm{D}_{1: \mathrm{n}}=$ dias para ocorrência da germinação.

\section{RESULTADO E DISCUSSÃO}

A porcentagem de germinação das sementes (Tabela 1) tratadas com $\mathrm{NaCl}$ e $\mathrm{CaCl}_{2}$, em todos os potenciais testados, diferiu estatisticamente em relação à testemunha, exceto no potencial de -0,2 MPa. Entretanto, as sementes tratadas com PEG apresentaram porcentagem de germinação significativamente menor em todos os potenciais testados, em comparação com a testemunha.

Com relação à velocidade de germinação, as soluções com polietileno glicol (PEG 6000) acarretaram menores valores em todos os potenciais (exceto a $0 \mathrm{MPa}$ ), em relação aos sais utilizados (Tabela 2).

\begin{tabular}{|c|c|c|c|}
\hline \multicolumn{4}{|c|}{ Germinação (\%) } \\
\hline \multirow{2}{*}{$\begin{array}{l}\text { Potenciais Osmóticos } \\
(\mathrm{MPa})\end{array}$} & \multicolumn{3}{|c|}{ Agentes Osmóticos } \\
\hline & PEG 6000 & $\mathrm{NaCl}$ & $\mathrm{CaCl}_{2}$ \\
\hline 0,0 & $96.0 \mathrm{Aa}$ & $96.5 \mathrm{Aa}$ & $96.0 \mathrm{Aa}$ \\
\hline$-0,2$ & $59.5 \mathrm{Bb}$ & $92.0 \mathrm{Aa}$ & $83.5 \mathrm{Aa}$ \\
\hline$-0,4$ & $27.5 \mathrm{Cb}$ & $49.5 \mathrm{Ba}$ & $61.0 \mathrm{Ba}$ \\
\hline$-0,6$ & $0.0 \quad \mathrm{Da}$ & $0.0 \mathrm{Ca}$ & $0.0 \quad \mathrm{Ca}$ \\
\hline$-0,8$ & $0.0 \quad \mathrm{Da}$ & $0.0 \mathrm{Ca}$ & $0.0 \quad \mathrm{Ca}$ \\
\hline $\begin{array}{l}\text { C.V. }(\%)=9,75 \\
\text { DMS potenciais }=1,01 \\
\text { DMS agentes }=0,86\end{array}$ & & & \\
\hline
\end{tabular}

Tabela 1 - Porcentagem de germinação de sementes Plantago ovata em diferentes potenciais e agentes osmóticos Table 1 - Percentage of germination of Plantago ovata seeds in different potentials and osmotic agents

Médias seguidas pela mesma letra maiúscula na coluna e minúscula na linha não diferem entre si, pelo teste de Tukey a 5\% de probabilidade.

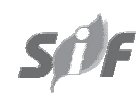

R. Árvore, Viçosa-MG, v.32, n.1, p.33-38, 2008 
Tabela 2 - Índice de velocidade de germinação de sementesde Plantago ovata em diferentes potenciais e agentes osmóticos

Table 2 - Index of speed of germination of Plantago ovata seeds, in different potentials and osmotic agents

\begin{tabular}{ccccc}
\hline \multicolumn{5}{c}{ Germinação (\%) } \\
\hline $\begin{array}{c}\text { Potenciais Osmóticos } \\
(\mathrm{MPa})\end{array}$ & \multicolumn{5}{c}{ Agentes Osmóticos } \\
\cline { 2 - 5 } & PEG & 6000 & $\mathrm{NaCl}$ & $\mathrm{CaCl}_{2}$ \\
\hline 0,0 & 48.0 & $\mathrm{Aa}$ & $48.0 \mathrm{Aa}$ & $48.25 \mathrm{Aa}$ \\
$-0,2$ & 14.71 & $\mathrm{Bb}$ & $20.04 \mathrm{Ba}$ & $21.45 \mathrm{Ba}$ \\
$-0,4$ & 6.21 & $\mathrm{Cc}$ & $14.04 \mathrm{Ca}$ & $9.83 \mathrm{Cb}$ \\
$-0,6$ & 0.0 & $\mathrm{Da}$ & $0.0 \mathrm{Da}$ & $0.0 \mathrm{Da}$ \\
$-0,8$ & 0.0 & $\mathrm{Da}$ & $0.0 \mathrm{Da}$ & $0.0 \mathrm{Da}$ \\
\hline
\end{tabular}

C.V. $(\%)=11,88$

DMS potenciais $=3,67$

DMS agentes $=3,13$

Médias seguidas pela mesma letra maiúscula na coluna e minúscula na linha não diferem entre si, pelo teste de Tukey a 5\% de probabilidade.

As sementes apresentaram pequena tolerância ao estresse hídrico simulado pelo PEG, com limite máximo entre -0,4 e -0,6 MPa, ressaltando-se que à partir de -0,6 e -0,8 MPa não houve germinação (Tabela 1).

Nas soluções de $\mathrm{NaCl}$ e $\mathrm{CaCl}_{2}$, o decréscimo significativo da germinação ocorreu com potenciais a partir de -0,4 MPa e com PEG a partir de -0,2 MPa, sendo a germinação totalmente inibida nos potenciais de -0,6 e -0,8 MPa, independentemente dos agentes osmóticos utilizados (Tabela 1). Entretanto, o estresse hídrico, como também o salino, causou redução significativa nos valores do IVG a partir do potencial -0,2 MPa (Tabela 2).

Os menores valores encontrados de porcentagem de germinação, quando os potenciais foram induzidos com PEG 6000 não concordam com os resultados de Khatri et al. (1991) e Fanti e Perez (1998), que verificaram maior efeito nas soluções salinizadas, quando comparadas com soluções osmóticas em potenciais equivalentes.

Reduções significativas da porcentagem e velocidade de germinação também foram observadas em sementes de Pterogyne nitens incubadas em soluções de PEG, sendo a germinabilidade afetada em potenciais hídricos a partir de -0,6 MPa e a velocidade de germinação, a partir de -0,4 MPa (NASSIF e PEREZ, 1997). Já as sementes de Adenanthera pavonina tiveram sua germinabilidade suprimida em soluções de PEG com potencial hídrico de $-0,5 \mathrm{MPa}$, mas a redução foi significativa em todos os potenciais testados, o mesmo ocorrendo com a velocidade de germinação (FANTI e PEREZ, 1998); e com relação a sementes de cássiado-nordeste houve aumento na porcentagem de germinação sob estresse hídrico e diminuição na velocidade de germinação, com o uso de PEG nos potenciais de -0,2 a -0,8 MPa (JELLER e PEREZ, 2003). Já nas sementes de Foeniculum vulgare a partir de -0,1MPa com PEG houve redução na germinação e vigor das sementes (STEFANELLO et al., 2006).

Sementes de diferentes espécies apresentam comportamentos semelhantes diante do estresse salino, diferindo no grau de tolerância aos sais. Como demonstrado em sementes de Leucaena leucocephala, o limite máximo de tolerância ao $\mathrm{NaCl}$ ocorreu a -1,3 MPa (CAVALCANTE e PEREZ, 1995); sementes de Copaifera langsdorffi apresentaram limites de -1,6 MPa para $\mathrm{NaCl}$ e -1,0 MPa para $\mathrm{CaCl}_{2}$ (JELLER e PEREZ, 1997); sementes de $P$. nitens suportaram a salinidade até os limites de -2,0 MPa e -1,6 MPa em soluções de

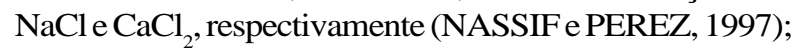
e sementes de Adenanthera pavonina tiveram limite máximo de tolerância de -1,4 MPa e -1,2 MPa, respectivamente nas mesmas soluções de $\mathrm{NaCl}$ e $\mathrm{CaCl}_{2}$ (FANTI e PEREZ, 1998).

Sementes de L. leucocephala também tiveram sua velocidade de germinação reduzida com o aumento da concentração de $\mathrm{NaCl}$ no meio germinativo (CAVALCANTE e PEREZ, 1995). O mesmo ocorreu com sementes de $P$. juliflora a partir do potencial de -0,3 $\mathrm{MPa}$ de $\mathrm{NaCl}$, enquanto em $\mathrm{CaCl}_{2}$ a redução só foi significativa a partir de -0,6 MPa (PEREZ e TAMBELINI, 1995). Sementes de $P$. nitens apresentaram redução na velocidade de germinação a partir dos potenciais de -0,8 e -0,4 MPa em $\mathrm{NaCl}$ e $\mathrm{CaCl}_{2}$, respectivamente (NASSIF e PEREZ, 1997); e nas sementes de Triticum aestivum a germinação e o índice de velocidade de germinação diminuíram com o aumento da concentração salina a partir de 45 e $60 \mathrm{mM}$ de $\mathrm{NaCl}$ (DUARTE et al., 2006); resultados semelhantes foram encontrados em Oryza sativa por Lima et al. (2005).

Os resultados indicaram que a velocidade de germinação das sementes de $P$. ovata foi mais afetada que a porcentagem de germinação. Tal fato vem corroborar a afirmação feita por Heydecker (1977) de que o aumento do estresse ambiental, em geral, leva inicialmente a um decréscimo na velocidade de germinação e só posteriormente vem afetar a germinabilidade das sementes. 
O efeito da salinidade na germinação não só dificulta a cinética de absorção da água, mas também facilita a entrada de íons em quantidade tóxica nas sementes em embebição (BRADFORD, 1995; BRACCINI et al., 1996). De acordo com Carvalho e Nakagawa (2000), o potencial hídrico afeta o alongamento celular e a síntese de parede, e para cada espécie existe um valor de potencial hídrico crítico, abaixo do qual a germinação não ocorre (CARVALHO, 2005). Pelos resultados deste trabalho, as sementes de Plantago ovata tiveram o seu desenvolvimento afetado pelo estresse hídrico, sendo o PEG 6000 o agente que reduziu mais a germinação da espécie.

\section{CONCLUSÃO}

Nas condições em que foi realizado este estudo, pode-se concluir que o:

Estresse hídrico induzido com PEG 6000 reduz a porcentagem e velocidade de germinação das sementes de Plantago ovata.

Estresse hídrico e salino afeta mais a velocidade do que a porcentagem de germinação.

O limite de tolerância das sementes ao estresse promovido por PEG, $\mathrm{NaCl}$ e $\mathrm{CaCl}_{2}$ está entre -0,4 e 0,6 MPa.

\section{REFERÊNCIAS}

BRACCINI, A. L. et al. Germinação e vigor de sementes de soja sob estresse hídrico induzido por soluções de cloreto de sódio, manitol e polietileno glicol. Revista Brasileira de Sementes, v.18, n.1, p.10-16, 1996.

BRADFORD, K. J. Water relations in seed germination. In: KIGEL, J.; GALILI, G. (Eds.). Seed development and germination. New York: Marcel Dekker, 1995. p.351-396.

BRASIL - Ministério da Agricultura e Reforma Agrária. Regras para análises de sementes. Brasília: SNDA/DNDV/CLAV, 1992. 365p.

CAMPOS, I. S.; ASSUNÇÃO, M. V. Estresse salino e hídrico na germinação e vigor do arroz. Pesquisa Agropecuária Brasileira, v.25, n.6, p.857-862, 1990.
CARVALHO, N.; NAKAGAWA, J. Sementes: ciência, tecnologia e produção. 4.ed. Jaboticabal: FUNEP, 2000. 588p.

CARVALHO, C. J. R. Respostas de plantas de Schizolobium amazonicum [S. parahyba var. amazonicum] e Schizolobium parahyba [Schizolobium parahybum] à deficiência hídrica. Revista Árvore, v.29, n.6, p.907-914, 2005.

CAVALCANTE, A. M. B.; PEREZ, S. C. J. G. A. Efeitos dos estresses hídrico e salino sobre a germinação de sementes de Leucaena leucocephala (Lam.) Pesquisa Agropecuária Brasileira, v.30, n.2, p.281-289, 1995.

DUARTE, G. L. et al. Physiological quality of wheat of seeds submitted saline stress.

Revista Brasileira de Sementes, v.28, n.1, p.122-126, 2006.

FANTI, S. C.; PEREZ, S. C. J. G. A. Efeitos do estresse hídrico, salino e térmico no processo germinativo de sementes de Adenanthera pavonina L. Revista Brasileira de Sementes, v.20, n.1, p.167-177, 1998.

HEYDECKER, W. Stress and seed germination: an agronomic view. In: KHAN, A. A. The

physiology and biochemistry of seed dormancy and germination. New York: North-Holland Publishing, 1977. p.237-282.

JELLER, H.; PEREZ. , S. C. J. G. A. Condicionamento osmático na germinação de sementes de cássia-do-nordeste sob estresse hídrico, térmico e salino. Pesquisa Agropecuária Brasileira, v.38, n.9, p.1025-1034, 2003.

KHATRI, R.; SETHI, V.; KAUSHIK, A. Interpopulation variations of $K$. indica during germination under different stresses. Annals of Botany, v.67, p.413-415, 1991.

LABOURIAU, L. G.; VALADARES, M. E. B. On the germination of seeds Calatropis procera (Ait.). Anais da Academia Brasileira de Ciências, v.48, n.2, p.263-284, 1976.

LARCHER, W. Ecofisiologia vegetal. São Carlos: Rima, 2000. 531p.

R. Árvore, Viçosa-MG, v.32, n.1, p.33-38, 2008 
LIMA, M. G. S. et al. Germinação e vigor de sementes de arroz submetidas a estresse salino.

Revista Brasileira de Sementes, v.27, n.1, p.54-61, 2005.

LIMA, J. D. et al. Efeito da temperatura e dos substrato na germinação de sementes de Caesalpinia ferrea Mart. ex. Tul. (Leguminosae)

Revista Árvore, v.30, n.4, p.513-518, 2006.

MAGUIRE, J. D. Speed of germination and in selection and evaluation for seedling emergence and vigor. Crop Science, v.2, n.1, p.176-177, 1962.

MAYER, A. M.; POLJAKOFF-MAYBER, A. The germination of seeds. 4.ed. Oxford: Pergamon Press, 1989. 270p.

MARTIN, H. H.; TRINE, E. Salinity tolerance during germination of seashore halophytes and salt-tolerant grass cultivars. Seed Science Research, v.15, n.1, p.43-50, 2005.

NASSIF, S. M. L.; PEREZ, S. C. J. G. A. Germinação de sementes de amendoim - do campo (Pterogyne nitens) influência dos tratamentos para superar a dormência e profundidade de semeadura. Revista Brasileira de Sementes, v.19, n.1, p.172-179, 1997.

PEREZ, S. C. J. G. A.; TAMBELINI, M. Efeito dos estresses salino e hídrico e do envelhecimento precoce na germinação de algarobeira. Pesquisa Agropecuária Brasileira, v.30, n.11, p.1289-1295, 1995.
REHMAN, S. et al. The effect of sodium chloride on germination and the potassium and calcium contents of Acacia seeds. Seed Science and Technology, v.25, n.1, p.45-57, 1996.

SALISBURY, F. B.; ROSS, C. W. Plant physiology. 4.ed. Belmont: Wadsworth Publishing Company, 1992. 682p.

SANTOS, V. L. M. et al. Efeito do estresse salino e hídrico na germinação e vigor de sementes de soja. Revista Brasileira de Sementes, v.14, n.1, p.189-194, 1992.

SILVA, J. G. et al. Seleção de 3 cultivares de algodoeiro para tolerância à germinação em condições salinas. Pesquisa Agropecuária Brasileira, v.27, n.9, p.1311-1317, 1992.

STEFANELLO, R. et al. Efeito da luz, temperatura e estresse hídrico no potencial fisiológico de sementes de funcho. Revista Brasileira de Sementes, v.28, n.2, p.135-141, 2006.

TOBE, K.; X. LI; OMASA, K. Effects of five different salts on seed germination and seedling growth of Haloxylon ammodendron (Chenopodiaceae) Seed Science Research, v.14, n.4, p.345-353, 2004.

VILLELA, F. A.; DONI-FILHO, L.; SEQUEIRA, E. L. Tabela de potencial osmótico em função da concentração de polietileno glicol 6000 e da temperatura. Pesquisa Agropecuária Brasileira, v.26, n.11/12, p.1957-1968, 1991. 